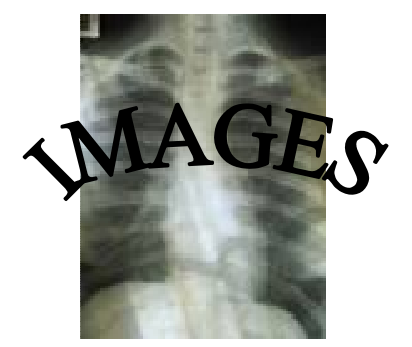

\title{
Chemotherapy-Induced Acral Erythema
}

\author{
Bassam I. Mattar, M.D. ${ }^{1,2}$
}

Bassem M. Chehab, M.D. ${ }^{2}$

${ }^{1}$ Cancer Center of Kansas, Wichita, KS

${ }^{2}$ University of Kansas School of Medicine-Wichita

Department of Internal Medicine

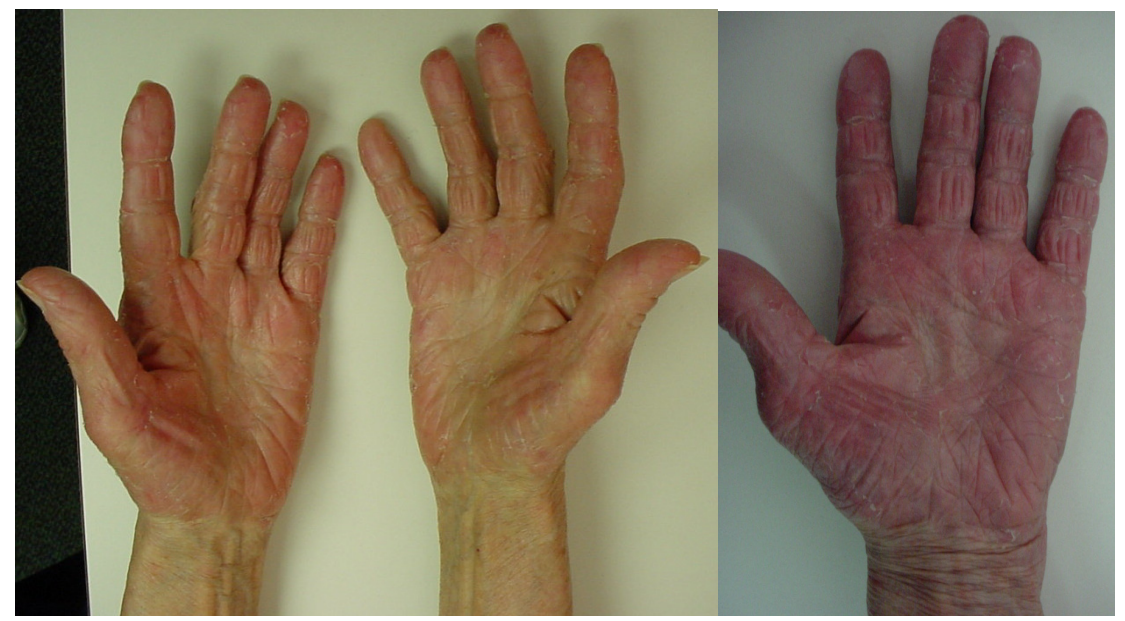

Cutaneous reactions to chemotherapeutics are common and may contribute significantly to the morbidity, and rarely to the mortality, of patients undergoing such treatments. Recognition and management of these reactions is important to provide optimal care. ${ }^{1}$ A 79-year-old woman was diagnosed with mycosis fungoides after a tissue biopsy of a lesion in her mid-back. She received vincristine and cytoxan chemotherapy. She presented with erythema, desquamation of both hands, and very stiff underlying dermis. Symptoms were thought to be due from her original disease of mycosis fungoides, but histological examination demonstrated vacuolar degeneration of the basal cell layer and spongiosis in the epidermis. The diagnosis was consistent with chemotherapy-induced acral erythema. Chemotherapy-related cutaneous toxicity includes generalized rashes such as the spectrum between erythema multiforme and toxic epidermal necrolysis, and site-specific toxicity such as mucositis, alopecia, nail changes, extravasation reactions, or hand-foot syndrome. Most of the toxicity is reversible with chemotherapy dose reductions or delays. Early recognition and treatment of the toxicity facilitates good symptom control, prevents treatment-related morbidity, and allows continuation of anti-cancer therapy. ${ }^{1-3}$

\section{References}

${ }^{1}$ Demircay ZG, Gurbuz O, Alpdogan TB, et al. Chemotherapy induced acral erythema in leukemic patients: A report of 15 cases. Int J Dermatol 1997; 36:593-598.

2 Tsuruta D, Mochida K, Hamada T, et al. Chemotherapy-induced acral erythema: Report of a case and immunohistochemical findings. Clin Exp Dermatol 2000; 25:386-388.

${ }^{3}$ Baack BR, Burgdorf WH. Chemotherapy-induced acral erythema. J Am Acad Dermatol 1991; 24:457-461.

Keywords: acral erythema, cutaneous, chemotherapy, case report 